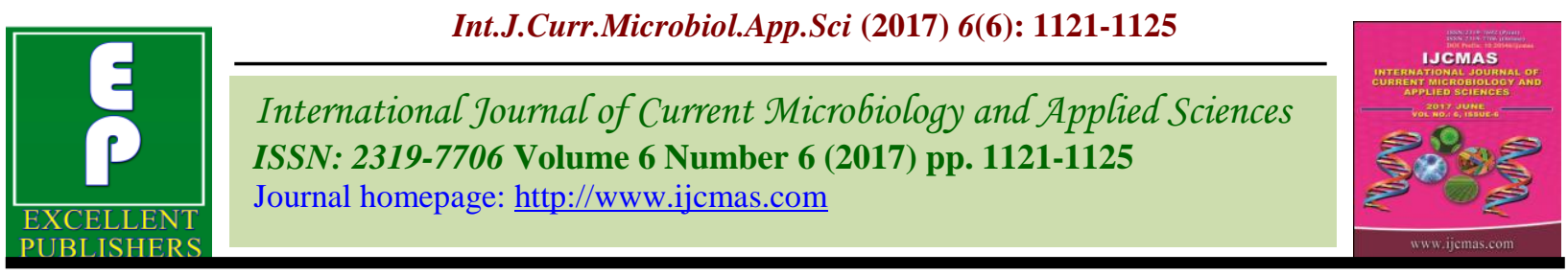

Original Research Article

https://doi.org/10.20546/ijcmas.2017.606.130

\title{
Bio-Efficacy of Insecticides against Diamond Back Moth Plutella xylostella (L.) in Cabbage (Brassica oleracea var. Capitata)
}

\author{
Anugu Anil Reddy*, Shashi Vemuri, CH. S. Rao and M. Aruna
}

Ainp on Pesticide Residues, Eei Premises, Rajendra Nagar, Hyderabad-500 030, A.P. India

*Corresponding author

\author{
A B S T R A C T
}

Keywords

Cabbage,

Insecticides,

Plutella xylostella

and Efficacy.

Article Info

Accepted:

17 May 2017

Available Online:

10 June 2017
A field experiment was conducted at student's farm, college of agriculture, Rajendranagar, Acharya N.G. Ranga Agricultural University, A.P during the year 2011- 12 in a randomized block design with seven insecticidal treatments which were replicated three times and tested against DBM (Plutella xylostella) on cabbage. Efficacy of seven insecticides viz., emamectin benzoate 5 SG at $11 \mathrm{~g}$ a.i.ha ${ }^{-1}$, emamectin benzoate $5 \mathrm{SG}$ at $22 \mathrm{~g}^{\text {a.i.ha }}{ }^{-1}$, profenophos $50 \mathrm{EC}$ at $500 \mathrm{~g}$ a.i.ha ${ }^{-1}$, profenophos $50 \mathrm{EC}$ at $1000 \mathrm{~g}$ a.i.ha ${ }^{-1}$, spinosad $45 \mathrm{SC}$ at $100 \mathrm{~g}$ a.i.ha ${ }^{-1}$, bifenthrin $10 \mathrm{EC}$ at $100 \mathrm{~g}$ a.i.ha ${ }^{-1}$ and Bacillus thuringiensis at $5 \mathrm{WP}$ at $25 \mathrm{~g}$ a.i.ha ${ }^{1}$ as evaluated during Kharif, 2012 against DBM (Plutella xylostella) on cabbage. Among all the insecticides, profenophos (1000 $\mathrm{g}$ a.i.ha $\left.{ }^{-1}\right)$ was found to be the most effective one with a maximum reduction in Plutella xylostella population (70.20\%), followed by bifenthrin $10 \mathrm{EC}$ at $100 \mathrm{~g}$ a.i.ha ${ }^{-1}(68.18 \%)$.

\section{Introduction}

Cabbage (Brassica oleracea var. capitata L.) is the second important cruciferous vegetable crop in the world. In India, it is cultivated in an area of $0.369 \mathrm{~m}$ ha with an average annual production of $7.949 \mathrm{~m} \mathrm{MT}$ and productivity of 21.5 MT $\mathrm{ha}^{-1}$. The major cabbage producing states are Maharashtra, Bihar, Karnataka, Orissa, West Bengal and Andhra Pradesh (NHB 2011). Cabbage contains adequate quantities of vitamins $\mathrm{A}, \mathrm{B}$ and $\mathrm{C}$ and minerals phosphorus, potassium, calcium, sodium and iron (Nath et al., 1984). Lack of quality seeds, improved cultivars, F1 hybrids and suitable production technology contribute partly to the lower yields, in addition to various other factors are responsible for the low productivity among which damage by various insect pests starting from transplanting till harvest is most significant.

A host of insect pests viz., diamond back moth, [Plutella xylostella (L.)], cabbage leaf webber, [Crociodolomia rinotalis (Zell.)], tobacco caterpillar, [Spodoptera litura(Fab.)] and mustard aphid, [Brevecornyae brassicae (L.)] etc., attack the crop. Among these diamond back moths, [Plutella xylostella (L.)] most notorious and pernicious pest on cruciferous vegetables causing 52 percent loss in marketable produce (Krishna kumar et al., 1986). Management of this pest has become a stupendous task and farmers apply pesticides 
8 to 10 times to effectively control this pest. The crop production strategies, now-a-days have however experienced a paradigm shift from pest "control" to pest "management". As exclusion of chemical insecticides is impracticable, the use of most selective and effective insecticide is essential. Hence experiments were conducted utilizing for their efficacy against DBM (P. xylostella).

\section{Materials and Methods}

The experiment was laid out in a Randomized Block Design (RBD) with 8 treatments including untreated control replicated thrice with individual plot size of $20 \mathrm{~m} 2(5 \mathrm{mx} 4 \mathrm{~m})$ and the insecticides viz., emamectin benzoate $5 \mathrm{SG}$ at $11 \mathrm{~g}$ a.i.ha ${ }^{-1}$, emamectin benzoate 5 $\mathrm{SG}$ at $22 \mathrm{~g}$ a.i.ha ${ }^{-1}$, profenophos $50 \mathrm{EC}$ at 500 g a.i.ha ${ }^{-1}$, profenophos $50 \mathrm{EC}$ at $1000 \mathrm{~g}$ a.i.ha , , spinosad $45 \mathrm{SC}$ at $100 \mathrm{~g}$ a.i.ha ${ }^{-1}$, bifenthrin $10 \mathrm{EC}$ at $100 \mathrm{~g}$ a.i.ha ${ }^{-1}$ and Bacillus thuringiensis at $5 \mathrm{WP}$ at $25 \mathrm{~g}$ a.i.ha ${ }^{-1}$ on cabbage first at head initiation and the second spray at ten days later were sprayed twice to evaluate the efficacy against Plutella xylostella.

The population of Plutella xylostella were recorded on five randomly selected plants in each head of the five selected plants in each plot. The percentage reduction at three, five and seven after each spraying were pooled and transformed into arc sine values which were further subjected to statistical analysis (Gomez and Gomez, 1984).

\section{Results and Discussion}

Bioefficacy results against Plutella xylostella (Tables 1 and 2) indicate that profenophos at $1000 \mathrm{~g}$ a.i.ha ${ }^{-1}$ followed by bifenthrin at $100 \mathrm{~g}$ a.i.ha ${ }^{-1}$ recorded high per cent reduction of $P$. xylostella population compared to emamectin benzoate $5 \mathrm{SG}$ at $11 \mathrm{~g}$ a.i.ha ${ }^{-1}$, emamectin benzoate $5 \mathrm{SG}$ at $22 \mathrm{~g}$ a.i.ha ${ }^{-1}$, profenophos 50
$\mathrm{EC}$ at $500 \mathrm{~g}$ a.i.ha ${ }^{-1}$, spinosad $45 \mathrm{SC}$ at $100 \mathrm{~g}$ a.i.ha ${ }^{-1}$ and Bacillus thuringiensis at $5 \mathrm{WP}$ at $25 \mathrm{~g}$ a.i.ha ${ }^{-1}$ in both sprays.

Data on over all efficacies (Table 1 and Fig. 2) of insecticides against Plutella xylostella after first spray revealed that all the insecticidal treatments were significantly superior over control. Profenophos at $1000 \mathrm{~g}$ a.i.ha ${ }^{-1}$ followed by bifenthrin at $100 \mathrm{~g}$ a.i.ha ${ }^{-1}$ were most effective recording 71.39 and $67.49 \%$ reduction of $P$. xylostella population. Emamectin benzoate at $22 \mathrm{~g}$ a.i.ha ${ }^{-1}$, profenophos at $500 \mathrm{~g}$ a.i.ha ${ }^{-1}$ and spinosad $100 \mathrm{~g}$ a.i.ha ${ }^{-1}$ recorded 62.59, 60.18 and $59.29 \%$ reduction of larval population but emamectin benzoate at $22 \mathrm{~g}$ a.i.ha ${ }^{-1}$ was on par with profenophos at $500 \mathrm{~g}$ a.i.ha ${ }^{-1}$ and spinosad at $100 \mathrm{~g}$ a.i.ha ${ }^{-1}$.

The data on overall efficacy (Table 2 and Fig. 2) revealed that all the insecticidal treatments were superior to control. Profenophos at 1000 $\mathrm{g}$ a.i.ha ${ }^{-1}$ was the most effective and superior to the remaining treatments recording $70.20 \%$ larval population reduction, followed by bifenthrin at $100 \mathrm{~g}$ a.i.ha ${ }^{-1}$ giving $68.18 \%$ larval population reduction and was superior over the other, viz., emamectin benzoate at 22 g a.i.ha ${ }^{-1}(61.97 \%)$, profenophos at $500 \mathrm{~g}$ a.i.ha ${ }^{-1}(60.00 \%)$, spinosad $100 \mathrm{~g}$ a.i.ha ${ }^{-1}$ $(59.07 \%)$ and emamectin benzoate at $11 \mathrm{~g}$ a.i.ha ${ }^{-1}(54.24 \%)$ reduction in larval population over control whereas, emamectin benzoate at $22 \mathrm{~g}$ a.i.ha ${ }^{-1}$ was on par with profenophos at (500 $\mathrm{g}$ a.i.ha $\left.{ }^{-1}\right)$ and spinosad $100 \mathrm{~g}$ a.i.ha ${ }^{-1}$. Bt $25 \mathrm{~g}$ a.i.ha ${ }^{-1}$ was found to be least effective in reducing the larval population $(40.75 \%)$.

The findings of the present study proved that profenofos at $1000 \mathrm{~g}$ a.i. $\mathrm{ha}^{-1}$ is an effective insecticide in controlling the Plutella xylostella. 
Table.1 Efficacy of insecticides against Plutella xylostella (L.) on cabbage after first spray

\begin{tabular}{|c|c|c|c|c|c|c|}
\hline \multirow[t]{2}{*}{ Treatment } & \multirow[t]{2}{*}{$\begin{array}{r}\text { Dosage } \\
\text { (g a.i./ha) }\end{array}$} & \multirow{2}{*}{$\begin{array}{c}\text { Pre-treatment } \\
\text { count } \\
\text { (number of } \\
\text { larvae / plant) }\end{array}$} & \multicolumn{4}{|c|}{$\begin{array}{l}\text { Mean per cent of reduction of larval } \\
\text { population over untreated check }\end{array}$} \\
\hline & & & 3 DAS & 5 DAS & 7 DAS & Over all \\
\hline $\begin{array}{l}\mathrm{T}_{1} \text { Emamectin Benzoate } \\
5 \% \mathrm{SG}\end{array}$ & 11 & 29.27 & $\begin{array}{l}49.80^{\mathrm{e}} \\
(44.80)\end{array}$ & $\begin{array}{l}53.84^{\mathrm{f}} \\
(47.20)\end{array}$ & $\begin{array}{l}59.38^{\mathrm{e}} \\
(50.41)\end{array}$ & $\begin{array}{l}54.34^{f} \\
(47.49)\end{array}$ \\
\hline $\begin{array}{l}\mathrm{T}_{2} \text { Emamectin Benzoate } \\
5 \% \mathrm{SG}\end{array}$ & 22 & 30.13 & $\begin{array}{l}56.75^{\mathrm{c}} \\
(48.88)\end{array}$ & $\begin{array}{l}63.03^{c} \\
(52.55)\end{array}$ & $\begin{array}{l}68.01^{\mathrm{c}} \\
(55.56)\end{array}$ & $\begin{array}{l}62.59^{c} \\
(52.29)\end{array}$ \\
\hline $\begin{array}{l}\mathrm{T}_{3} \text { Profenophos } \\
50 \% \text { EC }\end{array}$ & 500 & 30.33 & $\begin{array}{l}56.25^{\text {cd }} \\
(48.15)\end{array}$ & $\begin{array}{l}59.91^{\text {cd }} \\
(50.72)\end{array}$ & $\begin{array}{l}64.38^{\mathrm{cd}} \\
(53.36)\end{array}$ & $\begin{array}{l}60.18^{\text {cd }} \\
(50.87)\end{array}$ \\
\hline $\begin{array}{l}\mathrm{T}_{4} \text { Profenophos } \\
50 \% \text { EC }\end{array}$ & 1000 & 30.26 & $\begin{array}{l}60.94^{\mathrm{a}} \\
(51.32)\end{array}$ & $\begin{array}{l}75.84^{\mathrm{a}} \\
(60.58)\end{array}$ & $\begin{array}{r}78.14^{\mathrm{a}} \\
(62.15)\end{array}$ & $\begin{array}{l}71.39^{\mathrm{a}} \\
(57.67)\end{array}$ \\
\hline $\mathrm{T}_{5}$ Spinosad $45 \% \mathrm{SC}$ & 100 & 29.40 & $\begin{array}{l}55.48^{\text {cd }} \\
(48.15)\end{array}$ & $\begin{array}{l}59.53^{\text {cde }} \\
(50.49)\end{array}$ & $\begin{array}{l}62.69^{\text {de }} \\
(52.35)\end{array}$ & $\begin{array}{l}59.29^{\text {cde }} \\
(50.35)\end{array}$ \\
\hline $\begin{array}{l}\mathrm{T}_{6} \text { Bifenthrin } \\
10 \% \mathrm{EC}\end{array}$ & 100 & 29.00 & $\begin{array}{l}60.19^{a b} \\
(50.88)\end{array}$ & $\begin{array}{l}68.51^{b} \\
(55.87)\end{array}$ & $\begin{array}{r}73.77^{b} \\
(59.21)\end{array}$ & $\begin{array}{l}67.49^{b} \\
(55.24)\end{array}$ \\
\hline $\begin{array}{l}\mathrm{T}_{7} \text { Bacillus thuringensis } \\
5 \% \mathrm{WP}\end{array}$ & 25 & 29.33 & $\begin{array}{l}38.06^{f} \\
(38.09)\end{array}$ & $\begin{array}{l}41.53^{g} \\
(40.12)\end{array}$ & $\begin{array}{l}45.17^{f} \\
(43.22)\end{array}$ & $\begin{array}{l}41.58^{g} \\
(40.15)\end{array}$ \\
\hline $\mathrm{T}_{8}$ Control & -- & 29.31 & $\begin{array}{c}0.00 \\
(0.00)\end{array}$ & $\begin{array}{c}0.00 \\
(0.00)\end{array}$ & $\begin{array}{c}0.00 \\
(0.00)\end{array}$ & $\begin{array}{c}0.00 \\
(0.00)\end{array}$ \\
\hline S.Em & -- & & 0.65 & 0.77 & 0.85 & 0.72 \\
\hline C.D at $5 \%$ & -- & & 1.97 & 2.34 & 2.59 & 2.19 \\
\hline C.V.\% & -- & & 2.73 & 3.00 & 3.15 & 2.83 \\
\hline
\end{tabular}

DAS - Days After Spraying; Figures in the parentheses are angular transformed values.

Fig.1 Efficacy of insecticides against Plutella xylostella after first spray

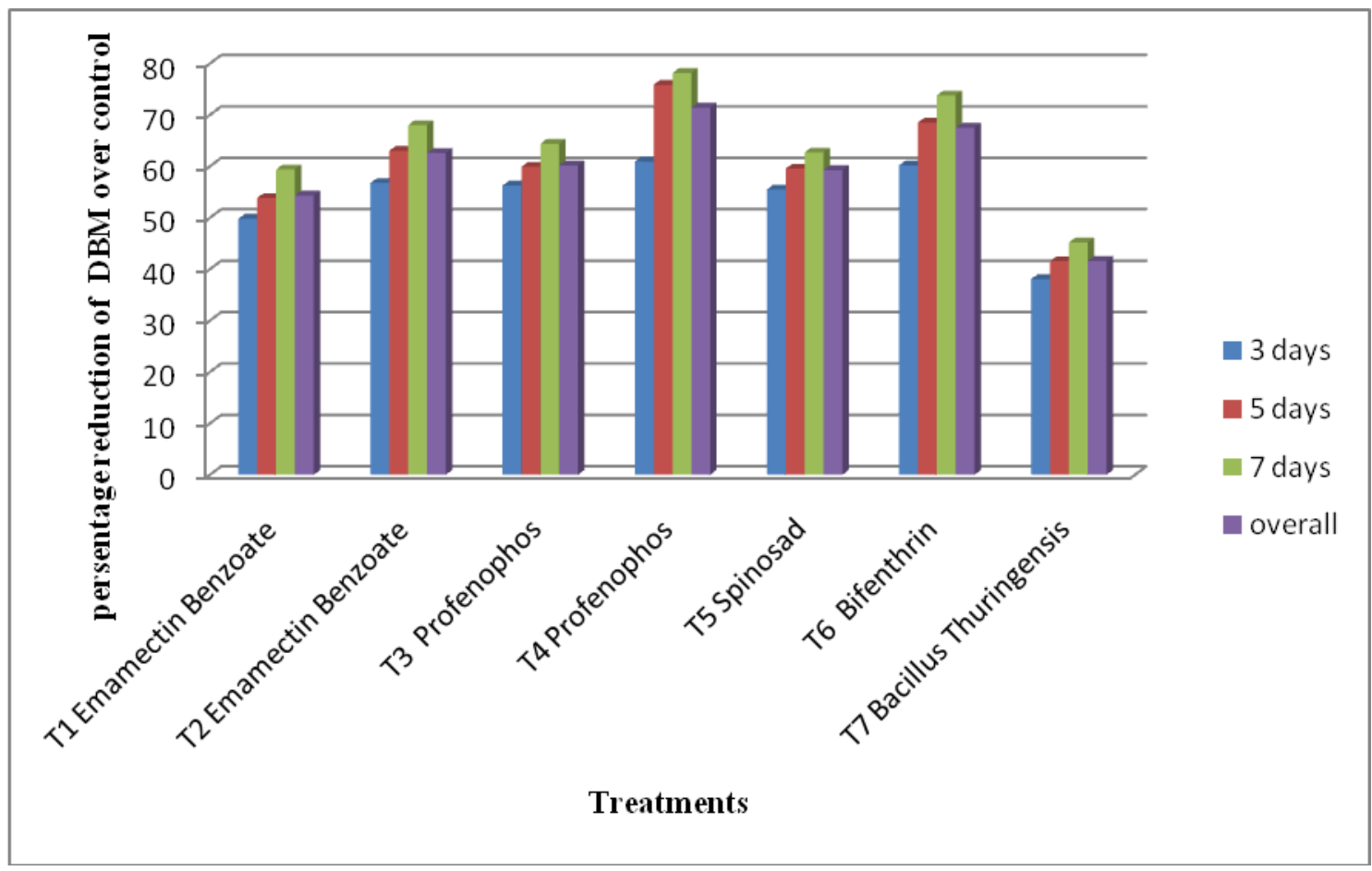


Table.2 Efficacy of insecticides against Plutella xylostella (L) on cabbage after second spray

\begin{tabular}{|c|c|c|c|c|c|}
\hline \multirow[t]{2}{*}{ Treatment } & \multirow[t]{2}{*}{$\begin{array}{c}\text { Dosage } \\
\text { (g a.i./ha) }\end{array}$} & \multicolumn{4}{|c|}{$\begin{array}{c}\text { Mean per cent of reduction of larval population } \\
\text { over untreated check }\end{array}$} \\
\hline & & 3 DAS & 5 DAS & 7 DAS & Over all \\
\hline $\begin{array}{l}\mathrm{T}_{1} \text { Emamectin Benzoate } \\
5 \% \mathrm{SG}\end{array}$ & 11 & $\begin{array}{l}46.22^{f} \\
(42.83)\end{array}$ & $\begin{array}{l}54.12^{\mathrm{ef}} \\
(47.36) \\
\end{array}$ & $\begin{array}{l}62.38^{\mathrm{ef}} \\
(52.17)\end{array}$ & $\begin{array}{c}54.24^{\mathrm{f}} \\
(47.43) \\
\end{array}$ \\
\hline $\begin{array}{l}\mathrm{T}_{2} \text { Emamectin Benzoate } \\
5 \% \mathrm{SG}\end{array}$ & 22 & $\begin{array}{l}57.18^{a} \\
(49.13)\end{array}$ & $\begin{array}{l}61.86^{\mathrm{c}} \\
(51.86)\end{array}$ & $\begin{array}{l}66.88^{c} \\
(54.87)\end{array}$ & $\begin{array}{l}61.97^{\mathrm{c}} \\
(51.93)\end{array}$ \\
\hline $\begin{array}{l}\mathrm{T}_{3} \text { Profenophos } \\
50 \% \text { EC } \\
\end{array}$ & 500 & $\begin{array}{c}54.50^{\text {abcd }} \\
(47.58)\end{array}$ & $\begin{array}{l}59.37^{\mathrm{cd}} \\
(50.40)\end{array}$ & $\begin{array}{l}66.14^{\text {cde }} \\
(54.42)\end{array}$ & $\begin{array}{l}60.00^{\mathrm{cd}} \\
(50.77)\end{array}$ \\
\hline $\begin{array}{l}\mathrm{T}_{4} \text { Profenophos } \\
50 \% \text { EC }\end{array}$ & 1000 & $\begin{array}{l}55.68^{a b c} \\
(48.26)\end{array}$ & $\begin{array}{l}72.76^{\mathrm{a}} \\
(58.55)\end{array}$ & $\begin{array}{l}82.12^{a} \\
65.03\end{array}$ & $\begin{array}{l}70.20^{\mathrm{a}} \\
(56.93)\end{array}$ \\
\hline $\mathrm{T}_{5}$ Spinosad $45 \% \mathrm{SC}$ & 100 & $\begin{array}{l}52.00^{\mathrm{de}} \\
(46.14)\end{array}$ & $\begin{array}{l}58.57^{\mathrm{de}} \\
(48.87)\end{array}$ & $\begin{array}{l}66.66^{\mathrm{cd}} \\
(54.74)\end{array}$ & $\begin{array}{l}59.07^{\text {cde }} \\
(50.23)\end{array}$ \\
\hline $\begin{array}{l}\mathrm{T}_{6} \text { Bifenthrin } \\
10 \% \mathrm{EC}\end{array}$ & 100 & $\begin{array}{l}57.08^{a b} \\
(49.07)\end{array}$ & $\begin{array}{l}69.48^{a b} \\
(56.48)\end{array}$ & $\begin{array}{l}78.00^{b} \\
(62.05)\end{array}$ & $\begin{array}{l}68.18^{a b} \\
(55.66)\end{array}$ \\
\hline $\begin{array}{l}\mathrm{T}_{7} \text { Bacillus thuringensis } \\
5 \% \mathrm{WP}\end{array}$ & 25 & $\begin{array}{l}28.01^{\mathrm{g}} \\
(31.95)\end{array}$ & $\begin{array}{l}40.50^{g} \\
(39.52)\end{array}$ & $\begin{array}{l}53.60^{\mathrm{g}} \\
(47.06) \\
\end{array}$ & $\begin{array}{l}40.75^{g} \\
(39.66)\end{array}$ \\
\hline $\mathrm{T}_{8}$ Control & -- & $\begin{array}{c}0.00 \\
(0.00)\end{array}$ & $\begin{array}{c}0.00 \\
(0.00)\end{array}$ & $\begin{array}{c}0.00 \\
(0.00)\end{array}$ & $\begin{array}{c}0.00 \\
(0.00)\end{array}$ \\
\hline S.Em & -- & 0.60 & 0.70 & 0.82 & 0.60 \\
\hline C.D at $5 \%$ & -- & 1.82 & 2.13 & 2.49 & 1.84 \\
\hline C.V.\% & -- & 2.65 & 2.75 & 2.91 & 2.39 \\
\hline
\end{tabular}

DAS - Days After Spraying. Figures in the parentheses are angular transformed values.

Fig.2 Efficacy of insecticides against Plutella xylostella after second spray

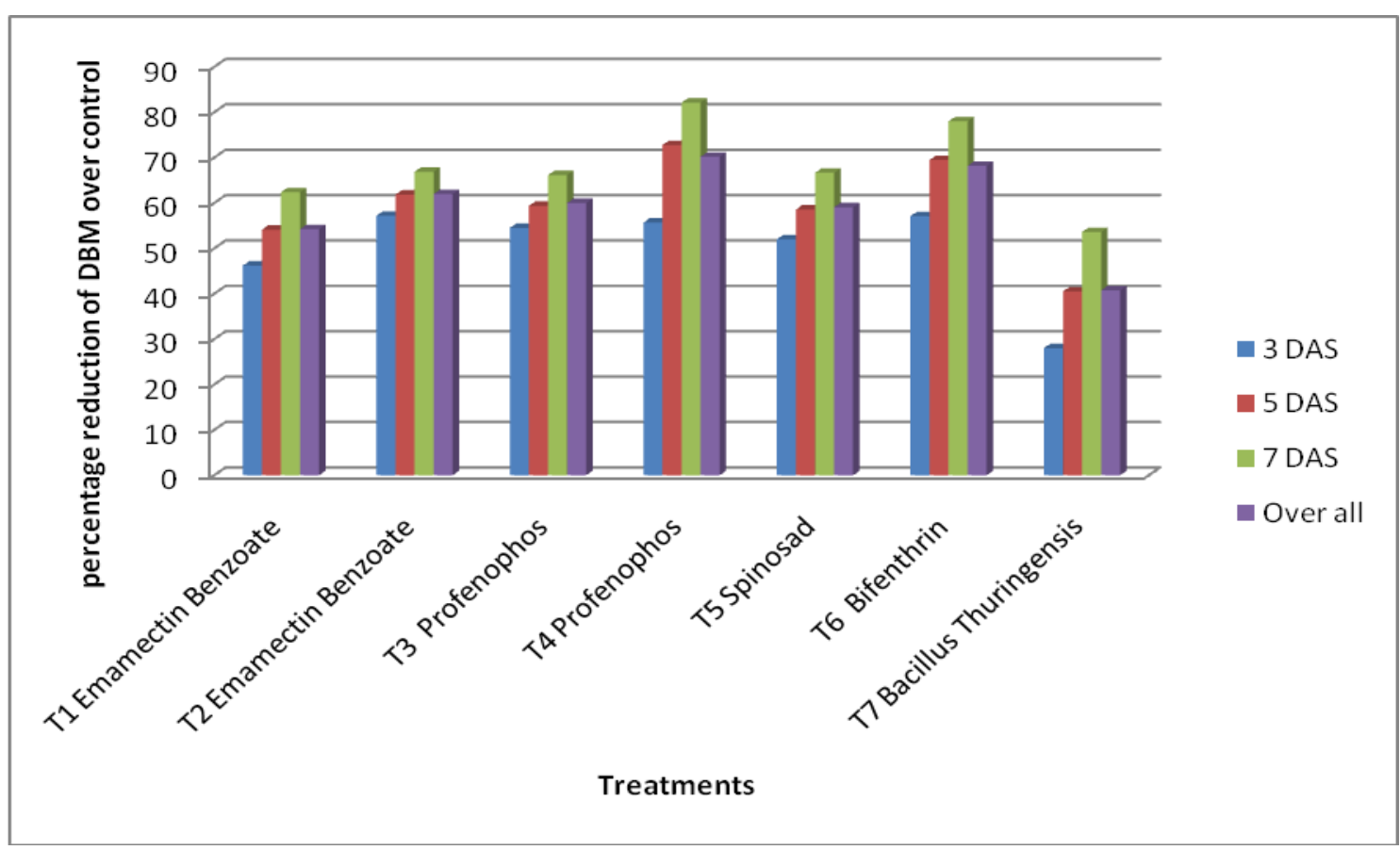


The performance of profenophos corroborate with the results of Williamson and Murray (1993) in suppressing $P$. xylostella with profenophos at 0.30 and $1.20 \mathrm{ml} \mathrm{lt}^{-1}$ at two weeks after spraying on cabbage. Profenophos at $0.25-0.5 \mathrm{~kg}$ a.i.ha ${ }^{-1}$ applied at 7-10 days intervals effectively reduced the larval population of $P$. xylostella on cabbage and Chinese kale (Calderson and Hare, 1986).

Murthy (1994) also obtained efficient control of $C$. binotalis with profenophos $(0.05 \%)$; profenophos was reported to be most effective against tobacco caterpillar at $0.05 \%$ (Prasad and Nandihalli, 1985); Murthy et al., (1997) reported profenophos @ $0.05 \%$ to be effective against $S$. litura at second day after spraying on cauliflower. Srikanth et al., (2000) reported that profenophos at $0.05 \%$ gave excellent control of C. pavonana (98.14\%) and S. litura $(80.06 \%)$ on cabbage.

\section{References}

Calderson, J.I. and Hare, C.J. 1986. Control of DBM in South East Asia by profenophos. Proc. First Int. Workshop, 289-295.

Gomez, K.A. and Gomez, A.A. 1984. Statistical procedures for Agricultural Research, Second edition. John willey and Sons, New York. pp. 582.

Krishna kumar, N.K. Srinivas, K. Suman, C.L. and Ramachander, P.R. 1986. Optimum control strategy of cabbage pests from a chemical control trial, Prog. Hort., 18: 104-110.

Murthy, B.N. 1994. Seasonal incidence of chemical control of pest complex of cauliflower, M.Sc. (Ag.) Thesis, ANGRAU, Hyderabad, pp 42.

Murthy, B.N. Rao, P.A. and Krishnaiah, P.V. 1997. Efficacy of different insecticides alone and in combinations with diflubenzuron against tobacco catterpillar $S$. litura on cauliflower. The Andhra Agri. J., 4: 59-61.

Nath, P. Velayudhan, S. and singh, D.P. 1984. Vegetable for the tropical region ICAR, Krishi Anusandhan Bhawan, Pusa, New Delhi.147.

National Horticultural Board. Annual report 2011. P. 184-187.

Prasad, N.K. and Nandihalli, B.S. 1985. Evaluation of certain insecticides for their efficacy and economics in the control of tobacco caterpillar, S. litura F., in VFC tobacco nursery field. Pesticides, 19: 34-36.

Sreekanth, M., Babu, T.R., Sultan, M.A. and Rao, B.N. 2000. Evaluation of certain new insecticides against lepidoptera pests of cabbage. Int. Pest Control, 42(4): 134-137.

Williamson, T.M. and Murray, 1993. Field evaluation of three insecticides at various application intervals for control of the DBM larvae and other pests of cabbage in Jamaica. Bulletin Research and Development Division, Ministry of Agriculture, Jamaica 68: 51-55.

\section{How to cite this article:}

Anugu Anil Reddy, Shashi Vemuri, Rao CH. S. and Aruna M. 2017. Bio-Efficacy of Insecticides against Diamond Back Moth Plutella xylostella (L.) in Cabbage (Brassica oleracea var. Capitata). Int.J.Curr.Microbiol.App.Sci. 6(6): 1121-1125.

doi: https://doi.org/10.20546/ijcmas.2017.606.130 